\title{
DESIGN AND IMPLEMENTATION OF AN AUTOMATED SPEECH RECOGNITION BASED TRANSPORT ENQUIRY SYSTEM
}

\section{SUNDEEP V V S AKELLA ${ }^{1}$, HEMANTH. ${ }^{2}$, KAKARLA AJAY KUMAR REDDY ${ }^{3}$, CH. VIJAYENDRA SAI ${ }^{4} \&$ PRASHANT R NAIR ${ }^{5}$}

${ }^{1,2,3,4}$ Department of Computer Science and Engineering, Amrita School of Engineering, Amrita Vishwa Vidhyapeetham, Tamil Nadu, India

${ }^{5}$ Associate Professor, Department of Computer Science and Engineering, Amrita School of Engineering, Amrita Vishwa Vidyapeetham, Tamil Nadu, India

ABSTRACT
The Voice-Based Public Transport Enquiry Systems are useful not only for providing the vehicle details but also to help
people who stand in long queues to get information about their journey details. This paper presents the design and
implementation details of a simple speech-recognition based system for the transport enquiry. This proposed system takes
the enquiry as voice input through the microphone embedded in the hardware. The voice is then analyzed and matched
with the details stored already in the transport database. The reply to the enquiry is given through display as well as
speech. This paper presents the design details of the proposed system which includes the architectural design, database
design and the output screen design. The benefit of the proposed system is also presented in this paper.
KEYWORDS: Voice-Recognition, Speech Analysis, Transport Enquiry, Text-To-Speech, Speech-To-Text

Received: May 09, 2020; Accepted: May 29, 2020; Published: Jun 30, 2020; Paper Id.: IJMPERDJUN2020187

\section{INTRODUCTION}

It is often experienced by many people waiting at a terminal for transport controllers to gather information about transportation convenience. There have been encounters many times where there will be no person giving this knowledge which clearly wastes time just to understand that, whether there is any convenience or not. The work presented in this paper is a solution to this problem. It reduces the human interference in presenting such information at the transport terminals. The aim of this work is to design and develop a voice-based automated transport enquiry system for bus transport. Speech Recognition (is also known as Automatic Speech Recognition $(A S R)$ ) is the method of changing a speech input into a sequence of meaningful words. Although much technological progress has been made in ASR, still there remains many research issues that need to be tackled.

The remaining part of the article designed as follows: Division 2 presents the related works, division 3 demonstrates the idea of the proposed system and the database design concepts and section 4 highlights the benefits of the proposed system. In division 5, sample results and the input screens related to the proposed system is described. Next, subdivision 6 concludes the paper.

\section{RELATED WORKS}

The works related to speech recognition and voice-controlled systems, in the literature, are briefed in this section. In the work presented in [1], the authors have explained about a programmable on-chip (PSoC) based embedded 
system which allows the user to use many options of speech recognition technology, the system proposed by the researchers is a perfectly constructed and easy to use programmable speech identification circuit. The authors in [2] introduced a voice-based transport enquiry system where the application software is deployed as a website in which the user provides the details from voice and the system provides the information through text. The interactive system presents options to update the system with new information like route information details and the timings for each route. The customer will be permitted to go through the obtained result. It also allows the user to move to the preceding and the succeeding sequence results through the speech instructions. The associated data also shall be displayed on the screen. This application-software works in a more interactive way compared to the others in the form of speech.

[3] Demonstrates a voice-based enquiry system that functions on the speech input given by the user. This system also uses speech-driven instructions as input by the user and gives the required data in the form of audio voice output. This system which is proposed by the researchers is implemented using .Net technology utilizing C\# programming language for front-end development, the system uses a SQL server for storing the information to be provided to the user and fetching it and uses the Raspberry Pi hardware to detect the voice from the user and uses the speech control mechanism to provide the voice output. Raspberry Pi is a major module in the system proposed and also would assist in converting the speech into text.

The work presented in [4] summarizes the full-length technological aspects and visualization of how speech to text conversion works and also gives a complete understanding of speech to text conversion based on Raspberry-Pi. A relative investigation of various methods proposed by different specialists is done according to stages. This work is achieved with the choice of the planned regulation for promoting strategy in human-computer interface operation in different mother-tongue and it also explains the several strategies utilized in each step of a speech recognition process and strives to innovate an approach for creating an adequate system for speech recognition.

The research work presented in [5] demonstrates that the work compromises with the design and implementation of Secure Home Automation using Raspberry Pi for mobile devices that leverage mobile technology to provide fundamental protection to our homes and associated control operations. Basically, the whole idea of developing this system is to automate the home using raspberry pi where the system can do all kinds of operations like ON/OFF, pause, resume. theses might be simple functions but are crucial when it comes to real-life applications, so here the developers have done a wonderful work by implementing this technology with mobile devices so that leverage mobile technology

In [6], researchers have explained a controlling device using voice recognition on the Matlab platform, they explained that Speech Recognition is the method of automatically identifying a certain speech addressed by a distinct speechmaker based on specific data included in speech waves. Here the system introduced makes it feasible to use the speaker's voice to verify his/her identification and implement controlled access to assistance like voice-based biometrics, database access services, voice-based dialing, voice mail and remote access to machines. In this design, the algorithms for speech identification have been generated and executed on MATLAB. These algorithms can be used for any security defense system in which the person's data privacy is challenged.

[7] Describes and states that Speech is the most leading \& primitive way of interaction amongst mankind. Nowadays Speech also has the capacity of being an essential way of interaction with machines. This article presents an outline of the Automatic Speech Recognition System, Division of Speech Recognition System and also includes a summary of the actions followed for generating the Speech Recognition System in stages. This writing also benefits in 
deciding the tool and procedure along with their relevant benefits \& defects. A relative examination of various methods is also covered in this journal.

The research presented in [8] elaborates on the effort to process the speech for a wide range of applications. the researchers say that Speech recognition and conversion to text are useful in the majority of the applications discovered these days. They quote that Speech has not much seen its past and present in the field of electronics and computers due to the complexity and variety of speech signals found and sounds. However, with modern methodologies, processors, complicated algorithms and techniques we can concoct speech signals to convert to text. This article deals with the appearance of text from a speech on a monitor employing an Android mobile, Bluetooth and Raspberry Pi. This application is quite beneficial in many places like classrooms and public transport areas. The researchers explain that speech-to-text conversion and presentation can also improve the system accessibility by providing data entry options for the physically handicapped users like blind, deaf. The code for an application program in raspberry pi is written employing Python programming language. AMR (Android Meets Robots) Voice application software is used for speech to text translation in android mobile. The speech given by the user is converted into a text file and the same text file is sent to Raspberry $\mathrm{Pi}$ through Bluetooth device which in turn is presented on the monitor. The application developed is currently the most useful and adaptable approach to speech conversion into text and display.

The article [9] gives a detailed description of speech to text conversion. In their project, they emerged with an online speech-to-text engine, executed as a system-on-a programmable-chip (SOPC) solution. They proposed a system that gets the speech while it is running through a microphone and analyzes the sampled speech to recognize the spoken words and text. They have made use of the Hidden Markov Model (HMM) for speech recognition. The obtained text can be stored in a file on a hard drive on a computer that connects to an FPGA that is placed on a development board using a standard RS-232 serial cable wire. The system can also supply other larger systems, giving users various choices for data entry.

The researchers, in [10], have composed this article for literature analysis. The essence is that Speech Recognition (SR) and its utilization are the fast-growing areas in research for the past three decades which are carried out on various features, notably in the domain of Information and Communication Technology (ICT) for speeding up scientific advancements. For effective and efficient communication between humans and machines, Automatic Speech Recognition (ASR) is currently employed.

[11] Explains an implementation of application software of the Voice Automated Transport Enquiry System. This system listens to the voice instructions given by the end-user and provides the desired data in speech. This application software can be effectively utilized in any transport terminal like Bus-stands, Railway terminals or Airports.

[12] Elaborates on the concept of automated speech identification Speech Recognition differs from Speaker recognition because it detects the person who wrote it the speech signal and is language freelance. Automatic Speech Recognition could be a technology that permits people at large to use their voices to talk with pc interfaces in an exceedingly approach that resembles a standard human voice communication. It allows users of data systems to speak entries rather than punching numbers on a keypad. The ASR system can be applied to various applications like conversational Automated Car Environment, Biomedical Applications, etc. A similar system of this type is presented in [13] also. 
Considering the different existing systems for the purposes of voice-based transport enquiry system, the presented system is designed and implemented to be a simple but effective one for this purpose. This system is implemented using React.js and Node.js. The ARTYOM.js speech recognition tool is integrated into this system. The required data are stored in a MySQL database.

Table 1: A Comparative Study on Existing and Proposed Model

\begin{tabular}{|l|l|c|c|c|}
\hline \multicolumn{1}{|c|}{ AUTHOR } & \multicolumn{1}{|c|}{ TITLE } & \multicolumn{1}{c|}{$\begin{array}{c}\text { SOFTWARE } \\
\text { ENVIRONMENT }\end{array}$} & $\begin{array}{c}\text { SPEECH } \\
\text { RECOGNITION: } \\
\text { Tool/Library }\end{array}$ & DATABASE : \\
\hline $\begin{array}{l}\text { Miss. Amruta P. } \\
\text { Deshmukh 1, Prof. } \\
\text { V. B. Bhagat 2, }\end{array}$ & $\begin{array}{l}\text { Voice Based } \\
\text { Retrieval For } \\
\text { Transport Enquiry } \\
\text { System }\end{array}$ & NET BEANS .NET & $\begin{array}{c}\text { Microsoft Speech } \\
\text { recognition } \\
\text { SDK }\end{array}$ & MySQL \\
\hline $\begin{array}{l}\text { Venkataramanan.S, } \\
\text { Suriya.D, Mr. } \\
\text { K.R.MohanRaj }\end{array}$ & $\begin{array}{l}\text { Voice Based } \\
\text { Transport Enquiry } \\
\text { System }\end{array}$ & ASP.NET & $\begin{array}{l}\text { Microsoft Speech } \\
\text { recognition SDK }\end{array}$ & MySQL \\
\hline Proposed System & $\begin{array}{l}\text { Voice-Based } \\
\text { Automated } \\
\text { Transport Enquiry } \\
\text { System }\end{array}$ & $\begin{array}{l}\text { REACT.JS } \\
\text { NODE .JS }\end{array}$ & ARTYOM & MySQL \\
\hline
\end{tabular}

\section{THE PROPOSED SYSTEM}

The proposed system maintains the details of the bus routes, passengers, tickets and other functionalities of the transport system. The whole system is operated using voice commands from the users. The voice commands given by the users are rerouted to the computerized automatic system and it gives a walkthrough to the users to question the important aspects of the bus and its detail. The first interaction with the system will be starting with a welcome greeting, the instructions (through speech) are presented to the user to tell about the source and destination locations of the travel. When the user makes his selection of the bus station/destination location the same is carried back and reinforced from the user. In case of any variation, the user is allowed to reselect the location(s). Once the specifications given by the user are verified, the system examines the database as per the specifications provided by the users. If relevant results are fetched from the database, the bus timings, the number of buses and service class details are provided to the user in the form of voice and display. The design of the proposed system includes the following modules

- Interface creation for master table management.

- $\quad$ Ticket and seat inquiry.

- Maintaining passenger reservation details.

- $\quad$ Preparing the route summary.

- Report generation.

- My SQL: is an open-source relational database management system.

- $\quad$ React JS: React could be a declarative, efficient, and versatile JavaScript library for building user interfaces. 
The architecture diagram of the proposed system is shown in Figure 1. The database is built using MySQL technology. The interactive web interface is made of react js, and the server connection to the database is given using node. The proposed system is implemented using react.js. The frontend (web-page) is developed using react and backend using node. The ARTYOM.JS (text-to-speech and speech-to-text) engines use a variety of methods to disambiguate the utterances. In a sound way, the meaning of the text is figured out and decided on the most appropriate meaning of the input in the given context. In administrator of this system authenticates the user, edit the database, create new timing and upload routes in the databases. The voice recognition component recognizes the commands given by the user

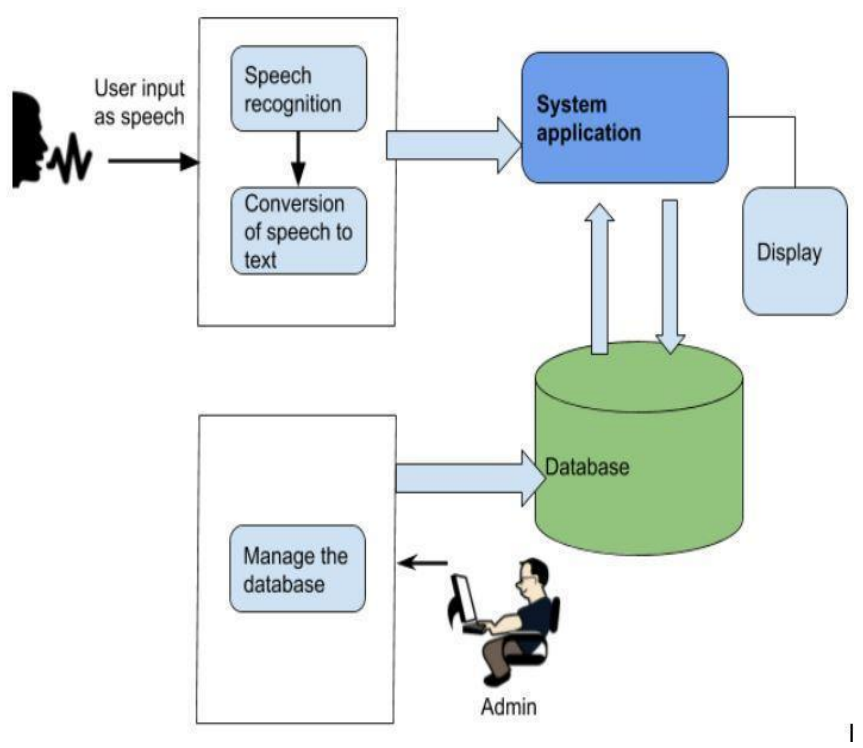

Figure 1: Architecture Diagram of the Proposed System.

\section{The Normalization Process of Database Design}

The database used is MySql and redundancy issues are removed by normalizing the database contents. The sequence steps followed at normalizing the database design is explained below.

\section{The Initial Schema}

The initial schema considering all the data elements involved in this system is given below Passengers(person_id,username,password,firstname,lastname,email,type,mobile,ticket_id ,timestamp,DOJ,bus_id,seat_no,bus_name,fare,arrival_time,departure_time,AC,bus_type ,total_seats,route_id,origin,destination,feedback_id,message,rating)

\section{Applying $1^{\text {st }}$ Normal Form}

Multi-valued attributes: seat_no.

- Passengers(person_id,username,password,firstname,lastname,email,type,mobile,ticket_id,timestamp,DOJ,bus_id, bus_date,bus_name,fare,arrival_time,departure_time,AC,bus_type,total_seats,route_id,origin,destination,feedbac k_id,message,rating)

- $\quad \operatorname{seats}($ ticket_id, $\underline{\text { seat_no }})$ 


\section{Functional Dependencies}

- $\quad$ username $\rightarrow$ \{person_id,password,firstname,lastname,email,type,mobile

- $\quad$ ticket_id $\rightarrow$ username,timestamp,DOJ,bus_id $\}$

- $\quad$ bus_id $\rightarrow\{$ bus_name,fare,arrival_time,

departure_time, AC, bus_type,total_seats,route_id\}

- $\quad$ route_id $\rightarrow\{$ origin,destination $\}$

- $\quad\{$ ticket_id,feedback_id $\} \rightarrow\{$ message,rating $\}$

\section{Applying $2^{\text {nd }}$ Normal Form}

\section{Candidate keys: \{ticket_id,feedback_id\}}

Passengers(person_id,username,password,firstname,lastname,email,type,mobile,ticket_id,timestamp,DOJ,bus_id,bus _name,fare,arrival_time,departure_time,AC,bus_type,total_seats,route_id,origin,destination,feedback_id,message,rating)

Decomposition of Passengers ():

- ticket_id $\rightarrow\{$ timestamp $\}$ is a partial dependency, so the table split into:

- Passenger_info(person_id,username,password,firstname,lastname,email,type,mobile, ticket_id,timestamp,DOJ,bus_id,bus_name,fare,arrival_time,departure_time,AC,bus_type,total_seats,route_id,ori gin,destination)

\section{Functional Dependencies}

- $\quad$ username $\rightarrow$ \{person_id,password,firstname,lastname,email,type,mobile $\}$

- ticket_id $\rightarrow$ username,timestamp,DOJ,bus_id $\}$

- bus_id $\rightarrow\{$ bus_name,fare,arrival_time,

departure_time,AC,bus_type,total_seats,route_id

- $\quad$ route_id $\rightarrow\{$ origin,destination $\}$

- feedback(ticket_id,feedback_id,message,rating)

- feedback_id, ticket_id $\rightarrow$ message, rating

\section{Applying $3^{\text {rd }}$ Normal Form}

- $\quad$ username $\rightarrow$ mobile,type,email,lastname,firstname, password,person_id

- $\quad$ ticket_id $\rightarrow$ bus_id,username,DOJ,timestamp

- bus_id $\rightarrow$ bus_name,fare,arrival_time,departure_time,AC,bus_type,total_seats,route_id,

- $\quad$ route_id $\rightarrow$ destination,origin

- feedback_id,ticket_id $\rightarrow$ rating,message 
All the above violates $3 \mathrm{NF}$

Decomposition of Passenger ():

- $\quad$ Person (username, mobile, type, email, last name, first name, password, person_id) by fd 1.

- Passenger_bus(username,ticket_id,timestamp,DOJ,bus_id,bus_name,fare,arrival_time,departure_time,AC,bus_typ e,total_seats,route_id,origin,destination)

Decomposition of Passenger_bus ():

- bus_route(bus_id,bus_name,fare,arrival_time,departure_time,AC,bus_type,total_seats,route_id,origin,destination) by Functional dependencies 3,4

- $\quad$ ticket(username,ticket_id,timestamp,DOJ,bus_id) by Functional dependency 2

Decomposition of bus_route():

- $\quad$ route(route_id,origin,destination) by functional dependency 4

- bus(bus_id,bus_name,fare,arrival_time,departure_time,AC,bus_type,total_seats,route_id) by functional dependency 3

\section{Applying BCNF}

- $\quad \operatorname{person}(\underline{\text { username}}$, mobile,type,email,lastname,firstname,password,person_id)

(username) $+=\{$ mobile,type,email,lastname,firstname, password,person_id $\}$

Therefore, it is in BCNF.

- route(route_id,origin,destination)

(route_id) $+=\{$ origin,destination $\}$ Therefore, it is in BCNF

- bus(bus_id,bus_name,fare,arrival_time,departure_time,AC,bus_type,total_seats,route_id)

(bus) $+=\{$ bus_id,bus_name,fare,arrival_time,departure_time,AC,bus_type,total_seats,route_id $\}$ Therefore it is in BCNF

- $\quad$ feedback(feedback_id,ticket_id,message,rating)

(feedback_id)+=\{ticket_id,message,rating $\}$ Therefore it is in BCNF.

- ticket(username,ticket_id,timestamp,DOJ,bus_id)

(ticket_id)+=\{username,timestamp,DOJ,b us_id $\}$.Therefore it is in BCNF.

- $\quad$ seats(ticket_id, seat_no $)$.It is in BCNF.

The schema design after normalization:

- $\quad \operatorname{person}(\underline{\text { username, }}$, mobile,type,email,lastname,firstname, password,person_id)

- $\quad \operatorname{route}($ route id, origin,destination)

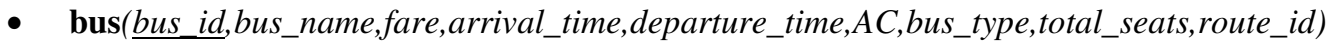


- $\quad$ feedback(feedback_id,ticket_id,message,rating)

- $\quad$ ticket(username,ticket_id,timestamp,DOJ,bus_id)

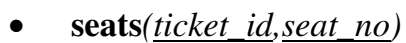

\section{BENEFITS OF THE PROPOSED SYSTEM}

This system is a needy practical method that is helpful in not only presenting the bus details, but also to help for travel planning and saves huge time for the user, which otherwise would have spent in waiting at the bus stations. This system works in a more interactive way with speech inputs. It needs less human intervention on the hardware of the system. It needs less maintenance and is much more efficient. Unlike with the traditional SMS based systems, in which the user needs to send SMS in predefined formats, this system is very simple to use and more accurate. Moreover, there is no requirement of human resources like in the enquiry desks. New stations and busses can be added easily and the details are readily available to the users of the system. It is also a low-cost system as no SMS gateway is required and no persons are required round the clock for running the system.

\section{FUTURE SCOPE}

With the ideology framed by the proposed system, in future the product can be extended to different kind of implementations for example: consider the same system for a airport kiosk which makes the work more easier and interactive, the speech synthesis can be given more support technically by training the product with different languages and slangs, this will help the product to be sustainable, to solve the issue for the social community.

\section{RESULTS AND DISCUSSIONS}

The home page designed for this system is shown in Figure 2. The registration form shown in Figure 3, allows two type of user-entries. One is the admin and the other one is the user/customer/consumer

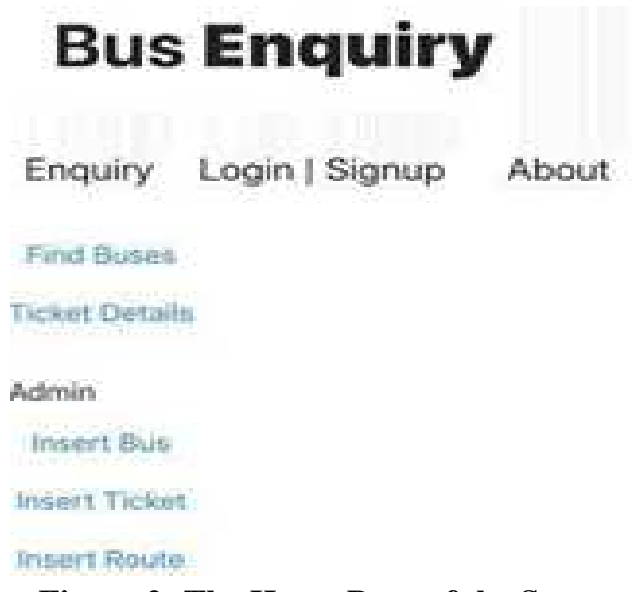

Figure 2: The Home Page of the System. 


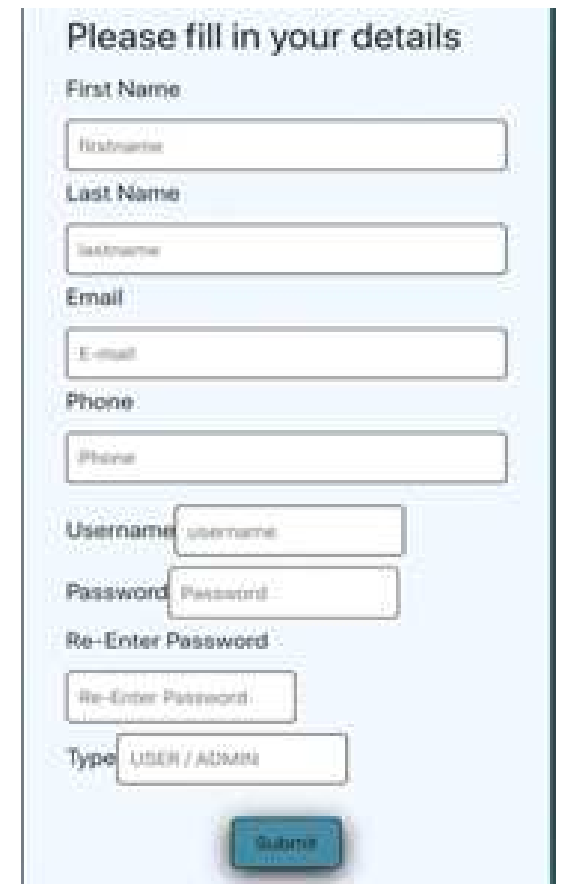

Figure 3: The Registration Form.

Enquiry Logh|S Sonve abova

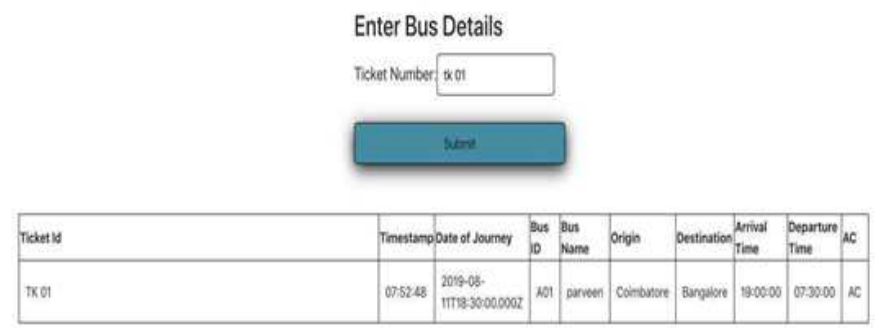

Figure 4: Displaying Ticket Details.

The screen designed for displaying the bus details for the given ticket number is shown in Figure 4. Here, when the user gives the ticket details through voice and says submit, then the system converts speech to text understands analyses the user's query, submits the details and refers the database according to the ticket number and reads out the displayed information on the screen.

In Figure 5, the system design for bus enquiry based on the journey plan of the passenger is shown Here, users can give the details of 'from journey' and 'to journey' through voice and submit. Then the system converts speech to text, analyses the user's query, submits the details and refers the database according to the journey details and displays the details of available buses with different arrival times and reads out the displayed information on the screen. 


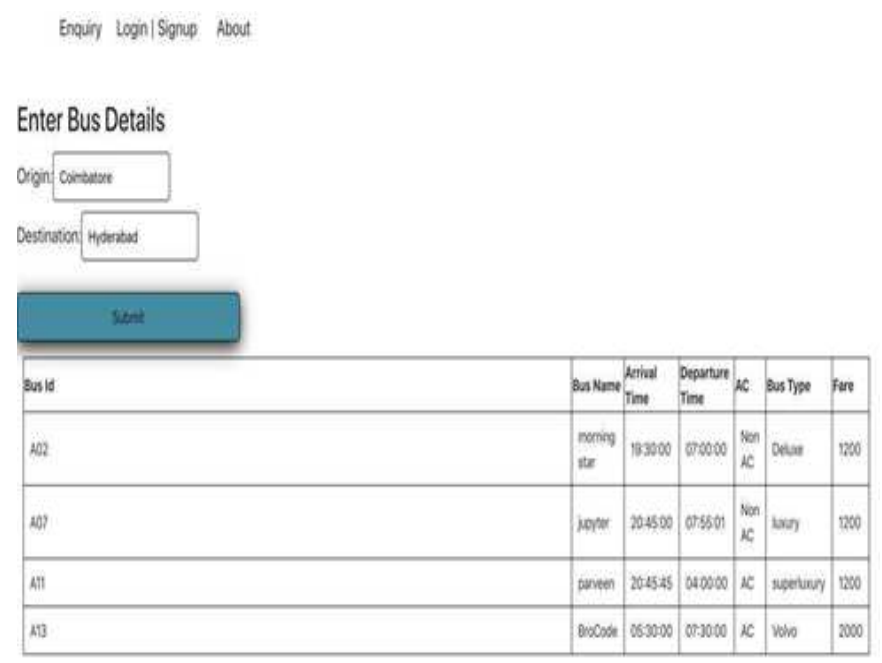

Figure 5: User Enquiry for Journey.

\section{CONCLUSIONS}

The design and implementation details of a simple Voice-Based Public Transport Enquiry System is presented in this paper. Unlike other SMS based systems and call enquiry based systems, in which the user needs to send SMS in predefined formats or he needs to call a number to get the information about his/her travel, this system is very simple to use and more efficient. Moreover, there is no requirement of human power like in the enquiry desks. New stations and busses can be added easily and the details are readily available to the users of the system. It doesn't require much cost to establish the system and, as no SMS gateway is required and no persons are required round the clock for running the system.

A big organization like Government running public transport service can provide toll-free numbers to the user and make the user happy with free calls to the system.

\section{REFERENCES}

1. Devarsh Barambe and S.P. Kodgire, “Speech recognition system for voice-controlled devices”, International Journal of Latest Trends in Engineering and Technology, Vol 6, No. 3, 2016.

2. Amruta P. Deshmukh and V. B. Bhagat "Voice-based retrieval for transport enquiry system", International Research Journal of Engineering and Technology, Vol. 06, No. 05, 2019.

3. Bitty Babu, Vaishali Pillai, Deeksha Shetty, and Savita Lohiya, Voice-Based Enquiry System Using Raspberry Pi, International Journal of Innovative Research in Science, Engineering and Technology, Vol. 5, No. 2, 2016.

4. Prachi Khilari and Bhope V, Implementation of Speech to Text Conversion, International Journal of Innovative Research in Science, Engineering and Technology, Vol. 4, No. 7, 2015.

5. S. Suresh and Y. Sindhuja Rao, Modelling of Secured Voice Recognition Based Automatic Control System, International Journal of Emerging Technology in Computer Science \& Electronics, Vol. 13, No. 2, 2015.

6. Sonam Kumari Kavita Arya and Komal Saxena, Controlling of Device Through Voice Recognition Using Matlab, International Journal of Advanced Technology \& Engineering Research, Vol. 2, No. 2, 2012.

7. Neerja Arora, Automatic Speech Recognition System: A Review, International Journal of Computer Applications, Vol. 151, No.1, pp. 0975-8887, 2016. 
8. M. Sudhakar Vandana Khareand, D Vijay Krishna Kanth, Speech to text conversion \& display using Raspberry Pi, IOSR Journal of Computer Engineering, Vol. 19, No. 4, pp. 14-18, 2017.

9. M.T. Bala Murugan and M. Balaji, SOPC-Based Speech-to-Text Conversion, Nios II Embedded Processor Design Contest, 2006.

10. Manjutha M, Gracy J, P Subashini and M Krishnaveni [4], Automated Speech Recognition System - A Literature Review, International Journal of Engineering Trends and Applications, Vol. 4, No. 2, 2017.

11. Amruta P. Deshmukh and V. B. Bhagat, Voice-Based Retrieval for Transport Enquiry System, International Research Journal of Engineering and Technology, Vol. 06, No. 05, 2019.

12. Pratiksha.C.Raut and Seema.U.Deogarh, Automatic Speech Recognition and its Applications, Vol. 03, No. $05,2016$.

13. Venkataramanan. S, Suriya. D, and K.R. Mohan Raj, "Voice-based transport enquiry system", International Journal of Advanced Networking \& Applications, Vol. 10, No. 5., 2019.

14. Tanksale, Pallavi R., and Snehal Kathale. "A Review of Wireless Home Automation System using Voice Recognition, GSM and A Web Portal Based on Zigbee." International Journal of Computer Science and Engineering (IJCSE) 3.3 (2014): $41-44$

15. Laxmi, H. Deepa, and B. Sujatha. "A Novel Approach to Lip Segmentation for Visual Speech Recognition." International Journal of Computer Science and Engineering (IJCSE) 5.2 (2016):57-62

16. Gupta, Umesh Kumar, and $R$ K. Prasad. "Frequency Analysis of Speech Signals for Devanagari Script Using Fft." International Journal of Electronics and Communication Engineering (IJECE) 2.3 (2013):41-48

17. Nagamani, M., et al. "Text-To-Speech System For Telangana State Languages." International Journal of Computer Science Engineering and Information Technology Research (IJCSEITR) 8.5 (2018):1-6

18. Lanjewar, Rahul B., and D. S. Chaudhari. "Comparative analysis of speech emotion recognition system using different classifiers on Berlin emotional speech database." Int. Journal of Electrical and Electronics Engineering Research (IJEEER). 3.5. (2013): 145-156.

19. Patel, Preeti. "Security of Information with Biometric Applications." IASET: International Journal of Library \& Educational Science (IASET: IJLES) 2.2 (2016):81-88 

